\title{
CREMONA INVOLUTIONS DETERMINED BY TWO LINE CONGRUENCES
}

\author{
EDWIN J. PURCELL
}

1. Introduction. Recently, in this Bulletin, ${ }^{1}$ we discussed a multiple null-correspondence formed by two line congruences. The first congruence consisted in the lines intersecting a fixed twisted curve of order $m$ and also its $(m-1)$-secant $d$, and the second congruence consisted in the lines intersecting another twisted curve $\delta_{n}^{\prime}$, of order $n$, and its $(n-1)$-secant $d^{\prime}$.

When $m=2$, the first curve is a conic $c_{2}$ having one point on $d$; a series of space Cremona involutions may now be defined as follows:

A generic point $P$ determines a ray $\rho_{1}$ of the first congruence and a ray $\rho^{\prime}$ of the second congruence. In the null-plane of $P$, formed by $\rho_{1}$ and $\rho^{\prime}$, lies another ray $\rho_{2}$ of the first congruence which intersects $\rho^{\prime}$ in $P^{\prime}$, the correspondent of $P$ in the involution.

2. The defining curves in general position. Let the equations of $d$ be $x_{1}=0, x_{2}=0$; and those of $d^{\prime}$ be $x_{3}=0, x_{4}=0$. Let the parametric equations of $c_{2}$ and of $\delta_{n}^{\prime}$ be, respectively,

$$
\begin{array}{ll}
x_{1}=\lambda^{2}, & x_{1}=f_{n}(s, t), \\
x_{2}=\lambda(\lambda-\mu), & x_{2}=F_{n}(s, t), \\
x_{3}=\lambda(\lambda-\mu), & x_{3}=\prod_{1}^{n-1}\left(t_{i} s-s_{i} t\right)(a s+b t), \\
x_{4}=\mu^{2}, & x_{4}=\prod_{1}^{n-1}\left(t_{i} s-s_{i} t\right)(c s+d t),
\end{array}
$$

where $s_{i}, t_{i}$ are the values of the parameters of $\delta_{n}^{\prime}$ at the $n-1$ points on $d^{\prime}$.

Then the equations of the involution are

$$
\begin{aligned}
& x_{1}^{\prime}=\left(x_{2}-x_{3}\right)\left(x_{1} F-x_{2} f\right)^{2}, \\
& x_{2}^{\prime}=\left(x_{1} F-x_{2} f\right) L, \\
& x_{3}^{\prime}=x_{3}\left\{\left(x_{2}-x_{3}\right)\left(x_{1} F-x_{2} f\right)\left(F-k H x_{2}\right)+\left(k H x_{1}-f\right) L\right\}, \\
& x_{4}^{\prime}=x_{4}\left\{\left(x_{2}-x_{3}\right)\left(x_{1} F-x_{2} f\right)\left(F-k H x_{2}\right)+\left(k H x_{1}-f\right) L\right\},
\end{aligned}
$$

where $f \equiv f_{n}\left(d x_{3}-b x_{4}, \quad a x_{4}-c x_{3}\right), \quad k \equiv(a d-b c), \quad F \equiv F_{n}\left(d x_{3}-b x_{4}\right.$,

${ }^{1}$ E. J. Purcell, $A$ multiple null-correspondence and a space Cremona involution of order $2 n-1$, this Bulletin, vol. 46 (1940), pp. 339-344. 
$\left.a x_{4}-c x_{3}\right), H \equiv \prod_{1}^{n-1}\left\{\left(s_{i} c+t_{i} d\right) x_{3}-\left(s_{i} a+t_{i} b\right) x_{4}\right\}, L \equiv\left(x_{2}-x_{3}\right)\left(x_{1} F-x_{2} f\right)$ $-\left(x_{1}-x_{2}\right)\left(k H x_{1} x_{3}-k H x_{2} x_{3}+F x_{3}-F x_{1}-f x_{3}+f x_{4}\right)-x_{1} x_{4}\left(F-k H x_{2}\right)$.

The order of the involution is $2 n+3$, where $n$ is any integer. The fundamental system and its principal images follow.

Line $d$ is a simple $F$-line of simple contact. The fixed tangent plane to all the homaloids is $x_{1}=0$, which is the plane through $d$ tangent to $c_{2} . d$ is of the first species and its $P$-surface is a ruled surface, $x_{1} F-x_{2} f=0$, of order $n+1$ formed by the rays of the second congruence which intersect $d$. Each such ray goes over into its point of intersection with $d$. On this $P$-surface, $d$ is a simple directrix line, $\delta_{n}^{\prime}$ is a simple directrix curve, and $d^{\prime}$ is an $n$-fold directrix line.

Line $d^{\prime}$ is a $2 n$-fold $F$-line without contact. It is of the first species. Its $P$-surface is of order $2 n+2$, whose equation is $\left(x_{2}-x_{3}\right)\left(x_{1} F-x_{2} f\right)\left(F-k H x_{2}\right)+\left(k H x_{1}-f\right) L=0$. On this $P$-surface $d$ is a simple line, $d^{\prime}$ is a $(2 n-1)$-fold line, $c_{2}$ is a simple conic, and $\delta_{n}^{\prime}$ is a double curve; by their intersections with these curves, all the $F$-lines of second species have more than $2 n+2$ points on the $P$-surface and therefore lie entirely on this surface.

Conic $c_{2}$ is a simple $F$-conic without contact. It is of the first species and has for $P$-surface the ruled surface of order $2 n+2$ formed by the rays of the second congruence which intersect $c_{2}$. Each such ray goes over into its point of intersection with $c_{2}$. On this $P$-surface $c_{2}$ is a simple directrix curve, $\delta_{n}^{\prime}$ is a double directrix curve, and $d^{\prime}$ is a $2 n$ fold directrix curve.

Curve $\delta_{n}^{\prime}$ is a double $F$-curve without contact. It is of the first species and has for $P$-surface $\left(x_{2}-x_{3}\right)\left(x_{1} F-x_{2} f\right) F-f L=0$, which is of order $2 n+2$. On this $P$-surface $d$ is a simple line, $d^{\prime}$ is a $2 n$-fold line, $c_{2}$ is a simple conic, and $\delta_{n}^{\prime}$ is a simple curve. Since $x_{1}=0$ is tangent to this surface along $d$, all the $F$-lines of second species lie on this $P$-surface.

The first is a $(1,2)$ congruence and the second is a $(1, n)$ congruence. There are thus $2 n+1$ common rays of both congruences. Each is a simple $F$-line of simple contact and is of the second species. The contact along these lines is torsal-that is, while all the homaloids have the same fixed tangent plane at a particular point on the line, the fixed tangent plane turns as this point moves along the line.

In the plane of $c_{2}$ are $n$ rays of the second congruence. Each is a simple $F$-line without contact and is of the second species.

The fixed tangent plane, through $d$, to all the homaloids contains $n$ rays of the second congruence. Each is a simple $F$-line without contact and is of the second species.

The locus of invariant points is a surface $\Sigma$ of order $n+3$. Its equa- 
tion is $x_{1} L-x_{2}\left(x_{2}-x_{3}\right)\left(x_{1} F-x_{2} f\right)=0$. On this surface $d$ is a double line, $d^{\prime}$ is an $n$-fold line, $c_{2}$ is a simple conic, and $\delta_{n}^{\prime}$ is a simple curve.

An alternate definition of the same involution employs this surface $\Sigma$. A generic point $P$ determines a ray $\rho^{\prime}$ intersecting $d^{\prime}$ once and $\delta_{n}^{\prime}$ once. Ray $\rho^{\prime}$ has with $\Sigma$ two in tersections, $\alpha$ and $\beta$, not on $d^{\prime}$ or $\delta_{n}^{\prime}$. We define $P^{\prime}$, the correspondent of $P$ in the involution, to be the harmonic conjugate of $P$ with respect to $\alpha$ and $\beta$.

3. Lower order for $d^{\prime}$ in special position. Any plane through $d^{\prime}$ intersects $\delta_{n}^{\prime}$ in one point $Z$ not on $d^{\prime}$.

If $d^{\prime}$ lies in the plane of $c_{2}$, this plane will contain such a point $Z$. Any line in the plane of $c_{2}$ passing through $Z$ is a ray of the second congruence. Each such line intersects $d^{\prime}$ once, $\delta_{n}^{\prime}$ once, and $c_{2}$ twice. It has $2 n+4$ intersections with every homaloid and therefore lies on every homaloid. The totality of these lines is the plane of $c_{2}$ which, being part of every homaloid, reduces the order of the involution by one.

The fundamental system is modified and the locus of invariant points is a surface of order $n+2$ on which $d^{\prime}$ is an $(n-1)$-fold line, $d$ is a double line, and $\delta_{n}{ }^{\prime}$ is a simple curve.

If the fixed tangent plane along $d$ to all the homaloids contains $d^{\prime}$, every line on it passing through $Z$ will lie on every homaloid. This plane will factor from the equations of the involution and their order will be one lower than in the general case.

Here the locus of invariant points is a surface, $\Sigma$, of order $n+2$ on which $d^{\prime}$ is an $(n-1)$-fold line, $\delta_{n}^{\prime}$ is a simple curve, $c_{2}$ is a simple conic; but $d$ does not lie on $\Sigma$.

4. The defining curves on one quadric surface. Let $d, d^{\prime}, c_{2}$, and $\delta_{n}^{\prime}$ lie on the same quadric surface, $d$ and $d^{\prime}$ being generators of the same system. Since $c_{2}$ intersects $d$ once, it intersects $d^{\prime}$ once. $\delta_{n}^{\prime}$ has $n-1$ points $\Delta_{i}^{\prime}$ on $d^{\prime}$ and therefore $n-1$ points $\Delta_{j}$ on $d$. It follows that $c_{2}$ and $\delta_{n}^{\prime}$ intersect in $n$ points. Every generator of the other system intersects $d, d^{\prime}, c_{2}$, and $\delta_{n}^{\prime}$, each once and thus has $1+2 n+1+2$ $=2 n+4$ intersections with every homaloid. Their locus is the quadric surface which is thus a part of every homaloid and factors from the equations of the involution, reducing the order by two. The involution in this case is of order $2 n+1$.

If the quadric surface is $x_{1} x_{3}-x_{2} x_{4}=0 ; d$ is $x_{1}=0, x_{2}=0 ; d^{\prime}$ is $x_{3}=0$, $x_{4}=0$; and the parametric equations of $c_{2}$ and $\delta_{n}^{\prime}$ are, respectively,

$$
x_{1}=\lambda \mu, \quad x_{1}=\prod_{n}^{2 n-2}\left(t_{j} s-s_{j} t\right) K(c s+d t)
$$




$$
\begin{array}{ll}
x_{2}=\lambda^{2}, & x_{2}=\prod_{n}^{2 n-2}\left(t_{j} s-s_{j} t\right) K(a s+b t), \\
x_{3}=\lambda \mu, & x_{3}=\prod_{1}^{n-1}\left(t_{i} s-s_{i} t\right)(a s+b t), \\
x_{4}=\mu^{2}, & x_{4}=\prod_{1}^{n-1}\left(t_{i} s-s_{i} t\right)(c s+d t),
\end{array}
$$

then the equations of the involution are

$$
\begin{aligned}
& x_{1}^{\prime}=K W\left(H x_{1}-K W x_{4}\right)\left(x_{1} x_{3}-x_{2} x_{4}\right), \\
& x_{2}^{\prime}=K^{2} W^{2}\left(x_{1}-x_{3}\right)\left(x_{1} x_{3}-x_{2} x_{4}\right), \\
& x_{3}^{\prime}=x_{3}\left(H x_{1}-K W x_{4}\right)\left(K W x_{1}-H x_{2}\right), \\
& x_{4}^{\prime}=x_{4}\left(H x_{1}-K W x_{4}\right)\left(K W x_{1}-H x_{2}\right),
\end{aligned}
$$

where $K$ is a constant, $H$ is as in $\$ 2$, and

$$
W \equiv \prod_{n}^{2 n-2}\left\{\left(s_{j} c+t_{j} d\right) x_{3}-\left(s_{j} a+t_{j} b\right) x_{4}\right\}
$$

In this involution, $d$ is a simple $F$-line without contact. It is of the first species and its $P$-surface is $x_{1} x_{3}-x_{2} x_{4}=0$.

$d^{\prime}$ is a $(2 n-1)$-fold $F$-line without contact. It is of the first species and its $P$-surface is $K W x_{1}-H x_{2}=0$.

$\delta_{n}^{\prime}$ is a simple $F$-curve of order $n$, of the first species, whose $P$-surface is $x_{1}=0$.

$\Delta_{j},(j=n, n+1, \cdots, 2 n-2)$, the intersections of $\delta_{n}^{\prime}$ and $d$, are isolated double $F$-points. Their $P$-surfaces are, respectively, $\left(s_{j} c+t_{j} d\right) x_{3}$ $-\left(s_{j} a+t_{j} b\right) x_{4}=0$.

$D_{1}, D_{2}, \cdots, D_{n}$, the $n$ intersections of $c_{2}$ and $\delta_{n}^{\prime}$, are isolated double $F$-points. The $P$-surface of each $D_{i}$ is the plane of that $D_{i}$ and $d^{\prime}$.

Point $R(0100)$, the intersection of $c_{2}$ and $d^{\prime}$, is a $2 n$-fold isolated $F$-point. Its $P$-surface is $H x_{1}-K W x_{4}=0$.

The $F$-curves of second species are as follows:

Lines $R \Delta_{j},(j=n, n+1, \cdots, 2 n-2)$, are simple $F$-lines of simple contact.

Lines $R D_{i},(i=1,2, \cdots, n)$, are simple $F$-lines without contact.

Through each $D_{i}$ there passes a single line intersecting both $d$ and $d^{\prime}$. Each is a simple $F$-line without contact.

The plane of $R$ and $d, x_{1}=0$, intersects the quadric surface in two lines, $d$ and $R S . x_{1}=0$ intersects $\delta_{n}^{\prime}$ in $n$ points, $n-1$ of which are the $\Delta_{j},(j=n, n+1, \cdots, 2 n-2)$, on $d$, and the remaining point is 
on $R S$. Thus $R S$ has $2 n$ points of intersection with every homaloid at $R$, one at $\delta_{n}{ }^{\prime}$, and one at $d$. Line $R S$ is a simple $F$-line without contact.

Since $Q(1000)$ lies on $d^{\prime}$, it is a $(2 n-1)$-fold point. But at $Q, n-1$ sheets of every homaloid have contact with the $n-1$ planes, $W=0$, through $Q$. The planes $W=0$ intersect $d$ in $\Delta_{j},(j=n, n+1, \cdots, 2 n-2)$. Each line $Q \Delta_{j}$ has $2 n$ points of intersection with every homaloid at $Q$ and two at $\Delta_{j}$. Lines $Q \Delta_{j}$ are simple $F$-lines without contact.

The locus of invariant points is $\Sigma$, a surface whose equation is $K x_{1} W\left(x_{1}-x_{3}\right)-x_{2}\left(H x_{1}-K W x_{4}\right)=0 . \Sigma$ is of order $n+1$ and contains $d^{\prime}$ as $(n-1)$-fold line without contact. $d$ is a simple line on $\Sigma$, but neither $\delta_{n}^{\prime}$ nor $c_{2}$ is on this surface. The alternate definition for this involution is: a generic point $P$ determines a ray $\rho^{\prime}$ intersecting $d^{\prime}$ once and $\delta_{n}^{\prime}$ once. Ray $\rho^{\prime}$ intersects $\Sigma n-1$ times on $d^{\prime}$, and in two other points, $\alpha$ and $\beta$, not on $d^{\prime} . P^{\prime}$, the correspondent on $P$ in the involution, is the harmonic conjugate of $P$ with respect to $\alpha$ and $\beta$.

5. The second congruence consists in the bisecants of a space cubic curve. We now consider the involution of order 9 that results when the second congruence consists in the bisecants of a twisted cubic curve, $q_{3}^{\prime}$.

Let $d$ be $x_{1}=0, x_{2}=0$. Let $c_{2}$ be $x_{1}=\lambda^{2}, x_{2}=\lambda \mu, x_{3}=\lambda^{2}, x_{4}=\mu^{2}$. Let the space cubic curve $q_{3}^{\prime}$ be $x_{1}=\sigma^{3}, x_{2}=\tau^{3}, x_{3}=\sigma \tau^{2}, x_{4}=\sigma^{2} \tau$. Then the equations of the involution are

$$
\begin{aligned}
& x_{1}^{\prime}=\left(x_{1}-x_{3}\right)\left(C^{2}-A B\right)^{2}, \\
& x_{2}^{\prime}=\left(C^{2}-A B\right) N, \\
& x_{3}^{\prime}=\left(x_{1}-x_{3}\right) A^{2}\left(C^{2}-A B\right)-B C N, \\
& x_{1}^{\prime}=B^{2} N-\left(x_{1}-x_{3}\right) A C\left(C^{2}-A B\right),
\end{aligned}
$$

where $A \equiv x_{3}^{2}-x_{2} x_{4}, B \equiv x_{4}^{2}-x_{1} x_{3}, C \equiv x_{1} x_{2}-x_{3} x_{4}$, and $N \equiv x_{1}^{3} x_{3}^{2}-x_{1}^{2} x_{3}^{3}$ $+x_{1}^{2} x_{2} x_{3} x_{4}+x_{1} x_{3}^{2} x_{4}^{2}+x_{2} x_{4}^{3}-x_{1}^{2} x_{2}^{3}-2 x_{2}^{2} x_{3}^{2} x_{4}+x_{2}^{3} x_{4}^{2}-x_{1} x_{2} x_{4}^{3}-x_{2}^{2} x_{4}^{3}-x_{1} x_{2} x_{3}^{3}$ $+3 x_{1} x_{2}^{2} x_{3} x_{4}+x_{1} x_{4}^{4}-2 x_{1}^{2} x_{3} x_{4}^{2}$.

The order of this involution is 9 .

In this involution, $d$ is a simple $F$-line of simple contact. It is of the first species and its $P$-surface, $C^{2}-A B=0$, is a ruled surface of order 4 consisting in the rays of the second congruence that intersect $d$. Each such ray goes over into its point of intersection with $d$.

$c_{2}$ is a simple $F$-conic of the first species whose $P$-surface is ruled and of order 8 , being formed by the rays of the second congruence that intersect $c_{2}$. 
$q_{3}^{\prime}$ is a fourfold $F$-cubic without contact. It is of the first species and its $P$-surface is of order 8.

Since the first congruence is of order 1 and class 2 and the second congruence is of order 1 and class 3 , there are 7 common rays of both congruences. Each such ray in tersects every homaloid 4 times at each of its two intersections with $q_{3}^{\prime}$, once on $d$, and once on $c_{2}$. Thus the seven common rays of both congruences lie on every homaloid. They are simple $F$-lines of simple contact.

The fixed tangent plane, $x_{1}=0$, through $d$ intersects $q_{3}^{\prime}$ in three points in general. The three lines joining these points two and two are rays of the second congruence lying in $x_{1}=0$. Each $\mathrm{such}$ ray intersects every homaloid 4 times at each of its two points on $q_{3}^{\prime}$ and twice on $d$. These three lines are simple $F$-lines without contact and are of the second species.

The plane of $c_{2}$ in general cuts $q_{3}^{\prime}$ in three points. There are thus three rays of the second congruence which cut $c_{2}$ twice. They are simple $F$-lines without contact and are of the second species.

$\Sigma$, the locus of invariant points, is $x_{1} N-x_{2}\left(x_{1}-x_{3}\right)\left(C^{2}-A B\right)=0$. This surface is of order 6 and contains $q_{3}^{\prime}$ as a double cubic curve. $d$ is a double line and $c_{2}$ is a simple conic on $\Sigma$.

Using this surface, we may define our involution: a generic point $P$ determines a bisecant $\rho^{\prime}$ of the cubic curve $q_{3}^{\prime}$. Ray $\rho^{\prime}$ intersects $\Sigma$ in two points, $\alpha, \beta$, not on $q_{3}^{\prime}$. $P^{\prime}$, the correspondent of $P$, is the harmonic conjugate of $P$ with respect to $\alpha$ and $\beta$.

When $d, c_{2}$, and $q_{3}^{\prime}$ lie on the same quadric surface, with $d$ intersecting $q_{3}^{\prime}$ in one point, all the generators of the other system drop away and the order of the involution is lower by two. A discussion similar to that in $\$ 5$ applies to this involution of order 7 .

University of Arizona 